\title{
Apolipoproteins and amyloid fibril formation in atherosclerosis
}

\author{
Chai Lean Teoh, Michael D. W. Griffin, Geoffrey J. Howlett ${ }^{\bowtie}$ \\ Department of Biochemistry and Molecular Biology, University of Melbourne, Parkville, Victoria 3010, Australia \\ Bio21 Molecular Science and Biotechnology Institute, University of Melbourne, Parkville, Victoria 3010, Australia \\ $\bowtie$ Correspondence: ghowlett@unimelb.edu.au \\ Received January 18, 2011 Accepted January 29, 2011
}

\begin{abstract}
Amyloid fibrils arise from the aggregation of misfolded proteins into highly-ordered structures. The accumulation of these fibrils along with some non-fibrillar constituents within amyloid plaques is associated with the pathogenesis of several human degenerative diseases. A number of plasma apolipoproteins, including apolipoprotein (apo) A-I, apoA-II, apoC-II and apoE are implicated in amyloid formation or influence amyloid formation by other proteins. We review present knowledge of amyloid formation by apolipoproteins in disease, with particular focus on atherosclerosis. Further insights into the molecular mechanisms underlying their amyloidogenic propensity are obtained from in vitro studies which describe factors affecting apolipoprotein amyloid fibril formation and interactions. Additionally, we outline the evidence that amyloid fibril formation by apolipoproteins might play a role in the development and progression of atherosclerosis, and highlight possible molecular mechanisms that could contribute to the pathogenesis of this disease.
\end{abstract}

KEYWORDS misfolding, apolipoproteins, amyloid fibril, atherosclerosis

\section{INTRODUCTION}

The function of most proteins depends upon the precise three-dimensional structure of their mature, folded form. How a newly synthesized chain of amino acids transforms itself into a perfectly folded protein depends both on the intrinsic properties of its amino acid sequence and on the cellular environment surrounding the polypeptide chain (Anfinsen, 1973). Ensuring accuracy in the protein folding process is crucial for maintaining proper cellular function. It is therefore inevitable that when proteins fail to fold correctly, or to remain correctly folded, malfunctioning of biological processes can arise leading to a wide variety of pathological conditions (Dobson, 2002). The number of diseases now known to be associated with protein misfolding is large, and particular attention has been paid to those in which misfolding results in the occurrence of a highly organized form of aggregates known as amyloid.

Amyloid deposits are composed predominantly of amyloid fibrils along with several non-fibrillar constituents such as lipids, proteoglycans, glycosaminoglycans and serum amyloid P component (SAP) (Alexandrescu, 2005; Pepys, 2006). To date there are over 25 proteins that accumulate as amyloid in human degenerative diseases, including Alzheimer's disease, Parkinson's disease, transmissible spongiform encephalopathies or prion diseases, and adult-onset (type II) diabetes. The ability to form amyloid is not restricted to proteins associated with amyloid deposits in vivo, as several proteins unrelated to disease have been shown to form fibrils in vitro (Chiti et al., 1999; Damaschun et al., 2000; Fändrich et al., 2001). In addition, some non-pathogenic and de novo synthesized peptides can also form fibrils that are morphologically and structurally indistinguishable from naturally occurring fibrils, leading to the idea that most, if not all polypeptide sequences, have the ability to form amyloid fibrils (Dobson, 2003).

Amyloid develops when proteins lose their native conformation and are converted into a predominantly $\beta$-sheet form, which increases their propensity to aggregate. Proteins that form amyloid fibrils are diverse, typically sharing little sequence or structural homology, but these proteins self associate to form ordered fibrillar structures that are remarkably similar. A number of plasma apolipoproteins are implicated in amyloid formation or influence the amyloid 
formation of other proteins. These include apolipoprotein (apo) A-I, apoA-II, apoC-II, apoE and the apolipoprotein-like proteins $\alpha$-synuclein and serum amyloid $A$ (Hatters and Howlett, 2002). This review will describe the occurrence of amyloid formation by apolipoproteins in disease, with particular focus on atherosclerosis. In addition, it will discuss recent in vitro studies which shed light on possible modulators of amyloid fibril formation by apolipoproteins, and will outline the evidence that amyloid fibril formation by apolipoproteins can play a role in the development of atherosclerosis.

\section{THE OCCURRENCE OF APOLIPOPROTEINS AS AMYLOID IN VIVO}

A number of plasma apolipoproteins are implicated in amyloidoses or co-localize to amyloid deposits in vivo. Apolipoproteins are over-represented among the proteins which form amyloid in various disease states (Sipe and Cohen, 2000). Apolipoproteins are an important class of serum proteins found embedded in the lipid surface of lipoprotein particles, such as low-density lipoprotein (LDL) and high-density lipoprotein (HDL). These protein-lipid complexes are responsible for the transport of triacylglycerols, cholesterol and phospholipids in the blood stream (LaRosa et al., 1970; Havel et al., 1973; Kinnunen et al., 1977). The susceptibility of apolipoproteins to amyloid formation may be attributed to their common structural and conformational properties (Hatters and Howlett, 2002). Apolipoproteins contain a high proportion of class $A$ amphipathic a-helical domains which mediate their binding to lipoprotein surfaces. The arrangement of these amphipathic $\alpha$-helices, which have a characteristic distribution of charged, hydrophilic, and hydrophobic residues, is believed to optimize the interaction with phospholipids (Segrest et al., 1994).

Apolipoproteins play a role in amyloid fibril formation by other proteins. The most extensive literature on apolipoproteins and amyloid has been that on apoE and the strong genetic linkage of apoE isoforms with Alzheimer's disease (Corder et al., 1993). ApoE exists as three common isoforms, which modulate the risk of developing Alzheimer's disease. Carriers of the $\varepsilon 4$ allele, encoding apoE4, are more susceptible to developing the disease, while those people carrying the $\varepsilon 2$ allele are less likely to develop the disease (Saunders et al., 1993; Frangione et al., 1996; Higgins et al., 1997; CedazoMínguez Cowburn, 2001). The mechanism of this susceptibility is not fully understood. Immunohistochemistry of amyloid deposits from Alzheimer's disease plaques shows co-localization of the C-terminal domain of apoE scattered throughout the fibril deposits (Castaño et al., 1995; Näslund et al., 1995). The C-terminal fragment of apoE binds to the $A \beta$ peptide and enhances its fibril formation (Strittmatter et al., 1993; Näslund et al., 1995; Permanne et al., 1997). ApoE has been identified immunohistochemically in many types of amyloid, suggesting that the influence of apoE may be more complicated rather than simply modulating amyloid formation by $A \beta$. Further, three separate studies have shown that the level of apoA-I in the serum of patients affected with neurodegenerative disease is reduced when compared to unaffected age matched individuals (Kuriyama et al., 1994; Kawano et al., 1995; Merched et al., 2000). Further longitudinal studies on apoA-I levels show that low apoA-I levels are a risk factor for the development of dementia (Saczynski et al., 2007).

In addition to the evidence that apolipoproteins contribute to amyloid disease indirectly, several apolipoproteins form amyloid in their own right (Higuchi et al., 1991b; Westermark et al., 1995; Wisniewski et al., 1995; Genschel et al., 1998; Hatters et al., 2000; Lange et al., 2000). There is no clear understanding of the molecular basis of the prominent role of apolipoproteins in many amyloidoses; however the low conformational stability of this family of proteins and the possibility of aberrant lipid interactions are proposed as potential factors involved (Hatters and Howlett, 2002). Apolipoproteins are considered prone to amyloid formation due to the dependence of their native conformation on the interaction with the lipoprotein surface (Chauhan et al., 1998; Acharya et al., 2002). Under lipid-free conditions, apolipoproteins have a diminished helical content and their conformational stability is significantly compromised leading to the development of an amyloidogenic conformation (Hatters and Howlett, 2002) and the formation of amyloid fibrils.

The appearance of apolipoprotein fibrils in vivo may result from mutations and truncations that reduce the ability of protein to bind to lipid surfaces (Andreola et al., 2003). Indeed, mutations in the N-terminal of apoA-I resulting in an increased local positive charge are associated with hereditary systemic apoA-l amyloidoses (Booth et al., 1995; Booth et al., 1996; Genschel et al., 1998), while mutations and truncations in the C-terminal of apoA-I are involved in the specific deposition of apoA-I in amyloidosis of the larynx, cardiac tissue, kidneys and cutaneous tissue (de Sousa et al., 2000; Benson et al., 2001). A total of 16 separate mutations in apoAI have been identified associated with the relatively rare systemic apoA-I amyloidoses (Eriksson et al., 2009). More commonly, apoA-I amyloid deposits are observed in the arterial intima and atherosclerotic plaque tissue (See section below). These deposits arise from wild-type apoA-I, reflecting the ability of the normal protein to form amyloid fibrils in vivo (Westermark et al., 1995; Obici et al., 2006). ApoA-II has also been reported to form amyloid in vivo in mice. One form of heritable senile amyloidosis in mice is linked to mutant forms of apoA-II that contain amino acid substitutions at four positions compared to non-amyloidogenic isoforms (Higuchi et al., 1991a). ApoA-Il has also been linked to amyloid deposition in humans. A patient with familial systemic amyloidosis in the kidney was found to have a 21 amino acid C-terminal extension of apoA-II as the predominant 
protein within the amyloid deposits (Benson et al., 2001). Sequencing the apoA-II gene confirmed the presence of a substitution mutation in the stop codon, resulting in the Cterminal extension of the protein sequence (Benson et al., 2001).

\section{IN VITRO CHARACTERIZATION OF APOLIPOPROTEIN FIBRIL FORMATION}

\section{ApoA-I}

ApoA-I is the major protein component of HDL. Previously, an $\mathrm{N}$-terminal fragment of apoA-I, which comprises residues 1-93, has been shown to aggregate in vitro to form fibrils at acidic pH (Andreola et al., 2003). However, systematic studies of the ability of full-length apoA-I to form amyloid fibrils in vitro have not been reported until recently (Wong et al., 2010). A range of solution conditions known to affect amyloid fibril formation in other protein systems were examined, and were not able to induce in vitro fibril formation by native apoA-I. The study showed that oxidation of the methionine residues of apoA-I is a key pre-requisite condition for the in vitro aggregation of lipid-free full-length apoA-I (Wong et al., 2010). Circular dichroism (CD) spectroscopy showed that oxidation of all three methionine residues in apoA-I caused partial unfolding of the protein and decreased its thermal stability, whereas analytical ultracentrifugation revealed that methionine oxidation inhibited the native self association of apoA-I to form dimers and tetramers. Methionine oxidized apoA-I aggregates show all defining characteristics of amyloid fibrils, displaying thioflavin T (ThT) reactivity as well as green-birefringence under cross-polarized light upon Congo Red (CR) binding. Examination of apoA-I aggregates by transmission electron microscopy (TEM) revealed fibrillar structures with a ribbon-like morphology, widths of approximately $11 \mathrm{~nm}$, and lengths of up to several microns (Fig. 1). X-ray fiber diffraction studies of the fibrils revealed a diffraction pattern with orthogonal peaks at spacings of $4.64 \AA$ and $9.92 \AA$, indicating a cross- $\beta$ amyloid structure. This systematic study of fibril formation by fulllength apoA-I represents the first demonstration that methionine oxidation can induce amyloid fibril formation (Wong et al., 2010).

\section{ApoE}

In vitro, a fragment of apoE which consists of amino acid residues 216-299 is able to form fibrillar structures (Wisniewski et al., 1995; Cho et al., 2001), whereas the full length protein has been shown to aggregate into amyloid-like fibrils under physiological salt and $\mathrm{pH}$ conditions (Hatters et al., 2006). It was observed that the rate of fibrillation of apoE was related to the conformational stability of the amino-terminal domain, with the E4 isoform of apoE forming fibrils the most readily, followed by E3 and E2. Characterization of apoE4 aggregates shows that they exhibit many properties of amyloid fibrils, including cross- $\beta$ structure, binding to the dyes ThT and CR, as well as fibrillar morphology. However, apoE4 aggregates differ from other amyloid fibrils in having fibrils which lack red-green birefringence when bound to $C R$. When viewed by TEM, the fibrillar morphology of apoE aggregates, while comparable in width $(14 \pm 6 \mathrm{~nm})$ to various amyloid fibrils of other proteins, is shorter in appearance and of less regular shape. CD analysis also shows that the secondary structure of apoE4 fibrils has a high apparent $\alpha$ helical content than $\beta$-structure. As apoE4 has a strong link to increased Alzheimer's disease risk, the finding that apoE forms fibrils and that E4 is the most fibrillogenic, and also displays the highest toxicity to neuronal cells, suggests a possible new mode of neurodegeneration that may be mediated directly by apoE (Hatters et al., 2006).

\section{ApoC-II}

ApoC-II is a 79 amino acid co-factor of the lipid-rich very low density lipoproteins and chylomicrons, which transport lipids through the bloodstream. Of all apolipoproteins, amyloid formation by apoC-II has been the most extensively characterized. Under lipid-free conditions in vitro, apoC-II spontaneously aggregates to form homogenous amyloid fibrils with all the hallmarks of amyloid fibrils (Hatters et al., 2000), including fibrous morphology (Fig. 1), the ability to interact with $C R$ and ThT, and cross- $\beta$ structure as shown by $X$-ray diffraction (Hatters et al., 2003). Short peptides corresponding to amino acids $56-76$ and $60-70$ of apoC-II also display the ability to self-assemble into fibrils in vitro and molecular dynamic (MD) simulations have been used to gain insights into the fibrillation properties of these small amyloidogenic peptides (Legge et al., 2007; Hung et al., 2008, 2009; Legge et al., 2009; Todorova et al., 2010).

The amyloid fibrils formed by a number of proteins typically take the form of twisted rope-like structures composed of several protofibrils. This results in a degree of morphological heterogeneity arising from fibrillar superstructures comprising variable numbers of protofilaments and variable degrees of twist and flexibility. In contrast, fibrils formed from lipid-free apoC-II are relatively homogenous in structure and form readily under quiescent conditions. This makes apoC-II a simple and convenient model system for the study of factors affecting the assembly and biophysical properties of amyloid fibrils in general. In particular, apoC-II amyloid fibrils are relatively soluble, allowing detailed biophysical analysis such as size distribution analysis using analytical ultracentrifugation (MacRaild et al., 2003; MacRaild et al., 2004b; Binger et al., 2008b; Mok et al., 2010). Extensive characterization of apoC-II amyloidogenic properties in vitro has provided much of the basis for our knowledge of amyloid fibril formation by apolipoproteins. 

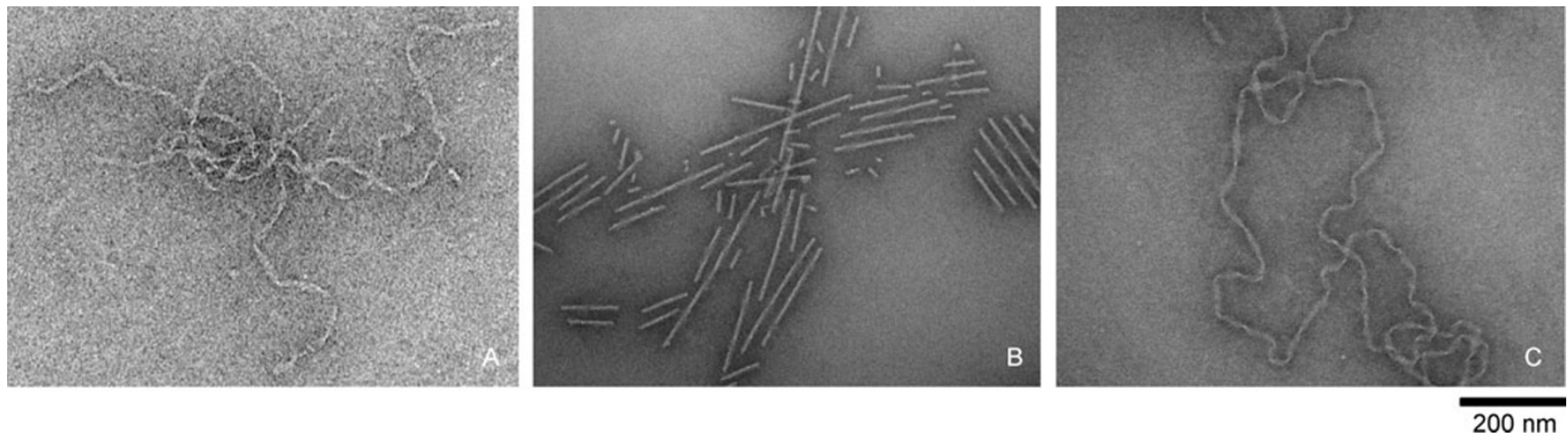

Figure 1. Apolipoprotein amyloid fibrils. Transmission electron micrographs showing the ultrastructural details of mature fibrils formed by (A) methionine oxidized apoA-I, (B) apoC-II in the presence of $500 \mu \mathrm{M}$ D9PC and (C) apoC-II alone. Adapted from (Griffin et al., 2008; Wong et al., 2010).

\section{Structural analysis of apoC-II amyloid fibrils}

The structure of apoC-II amyloid fibrils has been probed by Xray diffraction (Hatters et al., 2003; Teoh et al., 2011a). The diffraction image of aligned apoC-II fibrils shows a classical cross- $\beta$ diffraction pattern dominated by perpendicular reflections on the meridional and equatorial axes, where the sharp meridional peak at $4.67 \AA$ indicates the spacing between $\beta$-strands within the fibrils (Teoh et al., 2011a). The strong equatorial peak centered on $9.46 \AA$ represents the average spacing between $\beta$-sheets in the fibril cross-section. The equatorial axis radial profile shows that this is the only unique and resolved reflection on this axis, suggesting that the defined structure across the fibril cross-section is simple and does not contain superstructure resulting from lateral packing of filaments (Teoh et al., 2011a).

A large amount can be inferred from microscopy analysis of apoC-II fibrils. TEM images of negatively stained apoC-II fibrils (Fig. 1) revealed long unbranched fibrils of twisted ribbon morphology with widths of approximately $120-130 \AA$ and a typical length of several microns or more (Hatters et al., 2000; Hatters et al., 2003; Teoh et al., 2011a). These ribbons display a regular twist and, unusually, a small number take the form of closed loops (Hatters et al., 2000; Hatters et al., 2003; Teoh et al., 2011a). The ability of apoC-II fibrils to form closed loop structures is indicative of the high flexibility of these fibrils. Both random walk and worm-like chain models have been used to examine the probability of fibrils of different length closing to form a loop. Both models approximate the distribution of loop lengths measured from electron micrographs (Hatters et al., 2003). Atomic force microscopy performed under aqueous conditions, where the sample remains fully hydrated and tip-sample adhesion effects are mitigated, indicated a modal thickness of $37 \AA$ for the height distribution of apoC-II amyloid fibrils (Teoh et al., 2011a). Examination of apoC-II fibrils using scanning transmission electron microscopy (STEM) indicated that the fibrils are composed of one apoC-II molecule per $4.7 \AA$ rise of the cross- $\beta$ structure (Teoh et al., 2011a). In addition, total internal reflection fluorescence microscopy using ThT fluorescence which has been used to directly observe fibril growth at the single fibril level (Ban et al., 2003; Ban et al., 2004; Ban et al., 2006a; Ban et al., 2006b; Yagi et al., 2007; Andersen et al., 2009; Ozawa et al., 2009; Yagi et al., 2010) has been successfully applied towards the visualization of polymorphism in apoC-II fibrils, as well as to yield essential information about the mechanism of fibrillation (Teoh et al. 2011b).

The ability of single cysteine substitution mutants to form cross-links when incubated as fibrils under oxidizing conditions is consistent with a parallel, in-register structural model for apoC-Il fibrils (Fig. 2), where each residue in the $\beta$-strand of one apoC-II monomer is aligned in-register with the corresponding residues of its two neighbors (Pham et al., 2002; Teoh et al., 2011a). As X-ray diffraction studies indicate that the $\beta$-strands of apoC-II fibrils are separated by $4.67 \AA$, the anti-parallel model, where apoC-Il is present as a $\beta$ hairpin structure with the two consecutive anti-parallel $\beta$ strands within one $\beta$-sheet (Pham et al., 2002), would be unable to accommodate the formation of disulfide cross-links.

Hydrogen/deuterium exchange and limited-proteolysis studies coupled with mass spectrometry have identified regions of apoC-II that are involved in hydrogen bonding within the $\beta$-sheets of amyloid fibrils to be between residues 19-37 and 57-74 (Wilson et al., 2007). Time-dependent changes in the fluorescence properties of the single tryptophan residue in apoC-II (W26) observed upon fibril formation by wild-type apoC-II indicate that W26 is buried within the amyloid fibril structure (Hatters et al., 2000; Teoh et al., 2011a), while fluorescence spectroscopy of 1,5-IAEDANS-labeled cysteine variants of apoC-II revealed residues which underwent the greatest change in hydrophobicity upon fibril formation (Teoh et al., 2011a). Fluorescence resonance energy transfer (FRET) analysis of apoC-II fibrils provided distance constraints for selected donor and 
acceptor pairs located within the fibrils (Teoh et al., 2011a).

Arising out of these studies, a simple 'letter G-like' $\beta$ strand-loop- $\beta$-strand model for apoC-II fibrils was developed (Teoh et al., 2011a) (Fig. 2). The sole W26 is internalized, whereas $\mathrm{K} 30$ and D69 are oriented towards each other to satisfy buried charge. The two $\beta$-strands encompassing residues 20 to 36 and 58 to 74 are in parallel register between monomers and also parallel to each other. Fully solvated all-atom MD simulations showed that the model contained a stable cross $\beta$ core with a flexible connecting loop devoid of persistent secondary structure (Teoh et al., 2011a). The time course of the MD simulations revealed that charge clusters in the fibril rearrange to minimize the effects of same-charge interactions inherent in parallel-in-register models (Teoh et al., 2011a).

\section{FACTORS AFFECTING APOLIPOPROTEIN FIBRIL FORMATION AND INTERACTIONS}

\section{Phospholipids}

Lipids and lipid metabolites present in amyloid deposits have the potential to affect several areas of amyloid metabolism including the formation, stability, morphology and toxicity of fibrils (Knight and Miranker, 2004; Zhang et al., 2004; Zhao et al., 2004; Bosco et al., 2006; Myers et al., 2006; Griffin et al., 2008). Phospholipids exert significant effects on apoC-II fibril formation. In the absence of lipid, apoC-Il lacks stable helical structure, and adopts a monomeric random coil conformation which can undergo a transition to cross- $\beta$ structure as the protein aggregates and forms fibrils (Hatters et al., 2000). Our previous studies on apoC-II showed that in the presence of high concentrations of lipid micelles, apoC-II adopts a predominantly $\alpha$-helical structure (MacRaild et al., 2001; MacRaild et al., 2004a), presumed to resemble the native state of apoC-II bound to lipoprotein particles. This stabilization of native structure in a lipid environment is a property of a number of amyloidogenic proteins including other apolipoproteins (Hatters and Howlett, 2002). In contrast, low concentrations of micellar phospholipids and lipid bilayers, while initially inhibiting fibril formation, ultimately induce a novel, straight rod-like morphology for apoC-Il fibrils (Griffin et al., 2008; Teoh et al. 2011b), which is distinct from the twistedribbon morphology observed for apoC-Il fibrils formed in the absence of lipids (Hatters et al., 2000) (Fig. 1). These studies with apoC-Il show that phospholipid complexes can change the structural architecture of mature fibrils and generate new morphologies with the potential to alter the in vivo behaviour of amyloid.

While high concentrations of micellar lipids stabilize $\alpha$ helical structures and prevent fibril formation (Griffin et al., 2008), submicellar phospholipids and oxidized cholesterol derivatives accelerate amyloid fibril formation (Stewart et al., 2007). The role of individual lipid molecules in apoC-II amyloid fibril formation has been further explored using the model short-chain phospholipid dihexanoylphosphatidylcholine (DHPC) (Hatters et al., 2001a; Griffin et al., 2008). The addition of DHPC below its critical micelle concentration (CMC) to apoC-II leads to an increased rate of fibrillogenesis (Hatters et al., 2001a; Griffin et al., 2008). These results indicated that individual lipid molecules play an important role in amyloid fibril self-assembly, where monomeric DHPC is

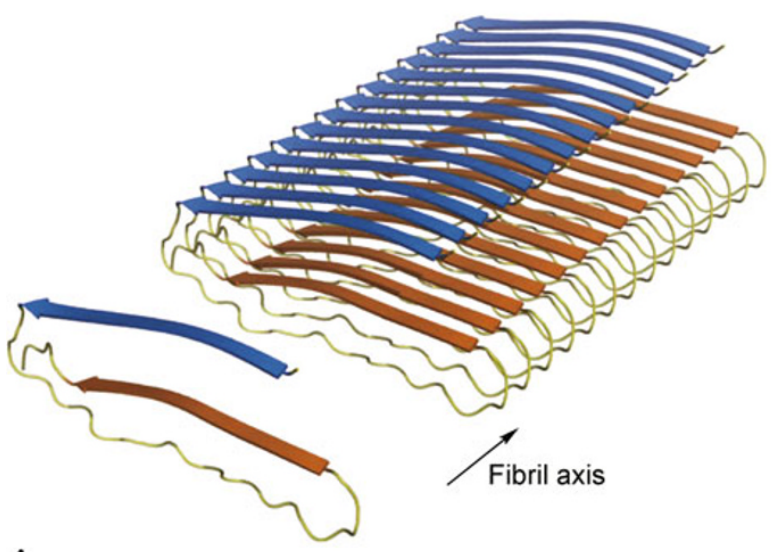

A

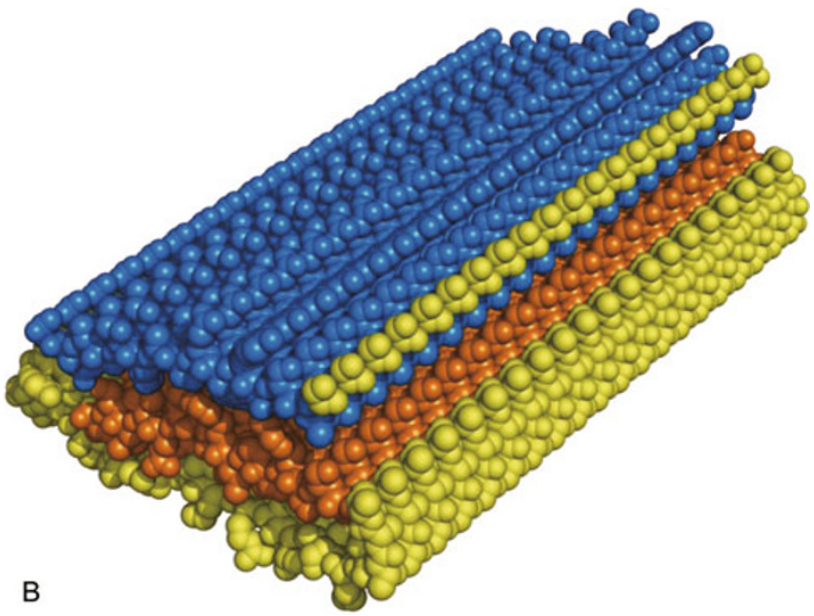

Figure 2. Structural model for apoC-II amyloid fibrils. (A) ApoC-II monomer assembles into amyloid fibrils with the described 'letter G-like' $\beta$-strand-loop- $\beta$-strand structure. The model includes residues $21-79$ of apoC-II and is shown as a side view, with each $\beta$-strand of the monomer contributing to each of the two $\beta$-sheets, giving rise to a parallel, in-register structure. The two $\beta$-strands of each monomer are also parallel to each other. (B) A space-filled view of the structural model for apoC-II ribbon-type amyloid fibrils. Adapted from (Teoh et al., 2011a). 
able to interact with apoC-II and accelerate amyloid formation (Hatters et al., 2001a). Further work demonstrated that activation of apoC-Il fibril formation occurs by promoting the rapid formation of a tetrameric species followed by a slow isomerization that precedes fibril growth (Ryan et al., 2008). This work also supports the distinct nature of nucleation and elongation, demonstrating that submicellar DHPC promotes the nucleation of apoC-II fibrils without significantly affecting the rate of fibril elongation or the rate of fibril breaking and joining (Ryan et al., 2010).

A range of amphipathic lipids and detergents were screened to systematically investigate the effect of lipid-like compounds on amyloid fibril formation by apoC-II (Ryan et al., 2011). The screen, conducted using a set of amphiphiles at half CMC, identified several activators and inhibitors. Further analysis revealed that all of the inhibitors induced the formation of apoC-II dimers enriched in a-helical content while the activators promoted the formation of stable apoC-II tetramers with increased $\beta$-structure. Addition of the test compounds after fibril formation had commenced allowed the effects of selected amphiphiles on fibril elongation to be assessed separately from their effects on fibril nucleation. The results indicated that specific amphiphiles induced structural changes in apoC-II that cause separate and independent effects on fibril nucleation and elongation (Ryan et al., 2011).

\section{Methionine oxidation}

The oxidation of proteins is associated with both natural aging and several disease processes (Stadtman and Levine, 2000). Methionine is one of the most easily oxidized amino acids, and its oxidation has been shown to modulate fibril assembly in several well-characterized amyloid systems. It was observed that preparations of apoC-II with both methionines oxidized were unable to form fibrils and that the position of oxidized methionine is important to its ability to inhibit fibrillogenesis (Binger et al., 2008a). Oxidation of the single methionine residue in the core fibril region of apoC-II (Met60) showed a greater effect on reducing the assembly of fibrils (Binger et al., 2008a). It was proposed that methionine residues are involved in specific hydrophobic interactions within the fibril core structure and that these are disrupted by the insertion of a single oxygen moiety, leading to the observed inhibition of fibril formation. Furthermore, oxidation of preformed apoC-Il fibrils dissociates the monomer in a time-dependent manner, indicating that changes in the chemical nature of the polypeptide chain in either the free monomeric pool or within the fibril can also have drastic effects on the stability of the mature fibrils (Binger et al., 2008a). Additional studies showed that oxidized methionines within apoC-II fibrils are resistant to reduction by methionine sulfoxide reductase enzymes (Binger et al., 2010).

Conversely, a recent study showed that oxidation of the methionine residues of apoA-I leads to aggregation of the protein into fibrils that display all the defining hallmarks of amyloid (Wong et al., 2010). In contrast to apoC-II, native, lipid-free apoA-I is relatively stable and does not form fibrils under a range of solution conditions. It was proposed that the oxidized methionines caused disruption of the native conformation and self assembly of the protein leading to fibril formation, but these residues may not be directly involved in hydrophobic packing within the fibril core. These results suggest that methionine oxidation may have a similar destabilizing effect on other amyloid forming apolipoproteins that have native structure, such as apoA-II. This is highlighted by the demonstration that methionine oxidation of apoA-II reduces the $\alpha$-helical nature of the protein (Anantharamaiah et al., 1988).

\section{Molecular chaperones}

The small-heat shock protein aB-crystallin acts as a chaperone by interacting with partially unfolded proteins to prevent their aggregation (Derham and Harding, 1999; Clark and Muchowski, 2000). aB-crystallin is over-expressed outside the lens in response to stress and is elevated in amyloid diseases, Alzheimer's disease, Parkinson's disease and Creutzfeldt-Jacob disease (Derham and Harding, 1999; Clark and Muchowski, 2000). aB-crystallin inhibits apoC-II amyloid formation at sub-stoichiometric concentrations (Hatters et al., 2001b). It is unlikely that aB-crystallin exerts this effect through interactions with the apoC-II monomer to stabilize it, since this type of interaction would require stoichiometric quantities of $\mathrm{aB}$-crystallin. Rather, $\mathrm{aB}$-crystallin may interact stoichiometrically with an oligomeric form of apoC-II which acts as a nucleus in the aggregation reaction. This oligomeric nucleus only exists transiently, since there is no detectable accumulation of intermediates in the fibrillogenesis of apoC-II (Hatters et al., 2001b). The transient nature of the nucleus allows aB-crystallin to act at sub-stoichiometric levels via a mechanism involving the conversion of this oligomeric species back to monomer, hence inhibiting amyloid formation (Hatters et al., 2001b). Alternatively, aBcrystallin may convert apoC-II monomers into conformations with a greatly reduced amyloidogenic propensity.

The extracellular chaperone clusterin co-localizes to amyloid deposits of atherosclerosis and Alzheimer's disease (May and Finch, 1992; Humphreys et al., 1999; Calero et al., 2000), and also inhibits fibril formation by apoC-Il at substoichiometric concentrations (Hatters et al., 2002). Clusterin has no effect on the sedimentation velocity behavior of preformed apoC-II fibrils, while apoC-II fibrils formed in the presence of clusterin show a reduced sedimentation coefficient distribution, indicative of reduction in the size of fibrils and/or a reduction in fibril-fibril interactions. These findings support the hypothesis that sub-stoichiometric levels of clusterin interact with the apoC-II nucleus converting it back 
to monomer, in a similar way to that proposed for the effect of aB-crystallin (Hatters et al., 2002).

\section{Serum amyloid P component and apolipoprotein E}

SAP is a circulating serum protein which co-localizes to all amyloid deposits that have been identified in vivo (Pepys et al., 1997; Kisilevsky, 2000). The finding that SAP mediates the deposition of amyloid fibrils and protects deposits from clearance (Tennent et al., 1995) has led to an interest in inhibitors of SAP-amyloid interactions, and to the suggestion of clearance of SAP for therapeutic purposes (Pepys et al., 2002).

The addition of SAP to solutions of pre-formed apoC-II fibrils increased their size distribution as determined by sedimentation velocity analysis (MacRaild et al., 2004b). These changes in the size-distribution of fibrils are much larger than expected for the binding of the SAP to the fibrils alone, suggesting that SAP increases fibril-fibril interactions and leads to a more tangled network structure. TEM of apoCII fibrils with SAP confirmed these findings (MacRaild et al., 2004b). We have used oscillatory rheometry to examine the viscoelastic properties of apoC-II fibrils in the presence and absence of SAP. Those studies showed that apoC-II fibrils behave as a weak gel in the absence of SAP, suggestive of an apoC-Il fibril network, which extends through the entire solution volume. In the presence of SAP, however, the gellike properties are lost, and the rheological behavior is suggestive of more localized network structure and a more colloid-like system (MacRaild et al., 2004b). The ability of SAP to tangle and condense amyloid fibrils may be an important factor in the effect of SAP on amyloid deposit stability.

ApoE influences the rate of amyloid formation by $A \beta$ peptide and a number of other amyloidogenic proteins (Evans et al., 1995; Soto et al., 1995; Naiki et al., 1997; Naiki et al., 1998 ) and is involved in the proteolytic clearance of amyloid fibrils by astrocytes (Koistinaho et al., 2004). Like SAP, apoE increases the size distribution of pre-formed apoC-II fibrils in a concentration-dependent manner, causing changes that are much greater than those calculated due to the added mass of the bound apoE (MacRaild et al., 2004b). These results suggest that apoE increases fibril-fibril interactions. This conclusion is further supported by TEM images, which showed a much more condensed apoC-II fibril network in the presence of apoE (MacRaild et al., 2004b). A later study observed that apoE binds to both apoC-II fibrils and $A \beta(1-40)$ fibrils with similar affinities via both the $\mathrm{N}$ - and $\mathrm{C}$-terminal domains, and undergoes similar conformational changes upon binding (Gunzburg et al., 2007). This suggests a common mechanism for the ability of apoE to cross-link amyloid fibrils, where the binding and cross-linking of amyloid fibrils is reliant upon independent interaction sites within the two domains of apoE.

\section{APOLIPOPROTEINS, AMYLOID FIBRILS AND ATHEROSCLEROSIS}

An age-related increase in localized amyloid deposits within the artery wall has been described. Amyloid deposits have been detected in the aortic media of $97 \%$ of atherosclerotic patients aged 50 years and over, and within the aortic intima of $35 \%$ of this same group (Mucchiano et al., 1992). Amyloid deposits in the media are most commonly derived from an integral fragment of smooth muscle cell-produced lactadherin termed medin (Häggqvist et al., 1999). However, amyloid deposits of heterogeneous origin have also been detected in the intima, where they are commonly associated with atherosclerotic plaques (Howlett and Moore, 2006). In fact, recent studies suggest that fibrillar amyloid species may be present in up to $60 \%$ of aortic atherosclerotic lesions (Mucchiano et al., 2001a; Mucchiano et al., 2001b; Röcken et al., 2006).

Many apolipoproteins, including apoA-I, apoA-II, apoB, apoC-Il and apoE (Westermark et al., 1995; O'Brien et al., 1998; Howlett and Moore, 2006), co-localize with amyloid deposits in atherosclerosis. Scant data exists, however, about the conformational states of most of the apolipoproteins found in arterial plaques, as immunologic reagents are not available to distinguish, in particular, fibrillar forms of apolipoproteins from their monomeric forms. At present, CR (Puchtler and Sweat, 1962) staining is the routine method applied for the detection of amyloid. However, $\mathrm{CR}$ has been shown to be unreliable in some cases, especially when only small amounts of amyloid are present (Westermark et al., 1999). New sensor dyes that are more sensitive and assay-friendly, which can stain biological tissues, have been sought. Previously, it was reported that a new group of styryl-based compounds have successfully penetrated the blood-brain barrier and stained for $\beta$-amyloid plaques specifically in vivo (Li et al., 2007).

Amyloid deposits in atherosclerosis appear to include amyloid fibrils derived from apoA-I (Westermark et al., 1995; Mucchiano et al., 2001a; Howlett and Moore, 2006). Despite being common, it is not known why apoA-I is deposited as amyloid specifically in atherosclerotic plaques; however these amyloid deposits are believed to be pathogenically linked to atherosclerosis progression. Substantial evidence indicates that oxidative processes and inflammation contribute to the pathogenesis of atherosclerosis (Ross, 1999; Chisolm and Steinberg, 2000). Inflammatory processes within atheroma lead to elevated levels of serum amyloid $A$ that can competitively displace apoA-I from HDL particles leading to increased local concentrations of lipid-free apoA-I (Mucchiano et al., 2001a), which, on its own, is known to increase the risk of amyloid formation. Furthermore, levels of myeloperoxidase and lipid hydroperoxides, both of which can cause specific oxidation of apoA-I methionines (Garner et al., 1998; Zheng et al., 2004), are greatly elevated within atherosclerotic 
tissue (Chisolm and Steinberg, 2000; Zheng et al., 2004). In addition to the total apoA-I concentrations that are already elevated, the proportion of methionine oxidized apoA-I is likely to be raised at these sites, where oxidation of methionine residues of apoA-I has been shown to be crucial for its fibrillation (Wong et al., 2010). Hence, the environment within atherosclerotic tissue would appear to favour conditions for methionine oxidation of apoA-I and fibril formation by the oxidized product.

ApoC-II accumulates extensively in atherosclerotic lesions of both murine and human arteries (Mak et al., 2002; Medeiros et al., 2004). Current immunohistological data suggests two distinct apoC-II populations within atherosclerotic plaques: one associated with peritoneal macrophages and the other co-localized with SAP, the marker for amyloid fibrils (Medeiros et al., 2004). Although usually associated with lipoprotein particles, apoC-II can also be secreted by macrophage foam cells and is detected in human and mouse atheroma (Mak et al., 2002; Medeiros et al., 2004). Oxysterols derived from oxidized LDL activate the nuclear receptors, liver $X$ receptor (LXR)- $\alpha$ and- $\beta$ that regulate expression of the apoE, C-I, C-II, and C-IV gene cluster (Mak et al., 2002). Expression of this gene cluster in lesional macrophage foam cells may increase secretion of lipid-free apolipoproteins into the sub-endothelial space where they would be susceptible to amyloidogenesis. In support of this, in human atherosclerotic lesions apoC-II co-localizes with SAP, a universal constituent of physiological amyloid deposits and is detected in the proximity of macrophage foam cells (Medeiros et al., 2004). Recently, SAP was shown to bind to fibrillar, but not monomeric apoC-II in vitro, suggesting that this acute phase reactant may modulate the fate of fibrillar apolipoproteins by limiting the availability of these cytotoxic fibrils to interact with cells in the artery wall (MacRaild et al., 2004b).

The pathological importance of amyloid deposits in atherosclerosis is highlighted by the findings that fibrils composed of apoC-II activate a pro-inflammatory macrophage signaling cascade mediated by the CD36 scavenger receptor (Medeiros et al., 2004), which is a key feature of vascular inflammation leading to lesion development. In contrast, oxidized LDL and non-fibrillar apoC-II failed to elicit the same effect, strongly indicating that activation of the macrophage response is dependent on amyloid species. This has led to the suggestion that the populations of apoC-II detected in atherosclerotic plaques may in fact be linked, where one population probably represents newly-synthesized apoC-II from macrophage foam cells, whereas the other population likely consists of fibrillar apoC-II accumulated from various sources such as the bloodstream. The finding that $A \beta$ amyloid also initiates macrophage inflammatory responses (Moore et al., 2002) suggests that this may be a general property of amyloid fibrils, including those composed of apoAI. It is tempting to further speculate that since activated macrophages over express apoC-II, an amplification cycle may develop in which the macrophage inflammatory response is continuously stimulated as more lipid-poor apoC-II is converted to amyloid fibrils.

Collectively, these combined factors may explain the common and specific association of apolipoproteins amyloid deposits with atherosclerotic plaque tissue. The majority of studies on mechanisms of atherosclerosis have hitherto focused on downstream lipids and inflammatory molecules that contribute to plaque formation. Further studies on the secondary structures of apolipopoproteins in atherosclerotic plaques, for example, may provide crucial insight into this potentially overlooked aspect of the atherogenic process.

\section{ABBREVIATIONS}

1,5-I-AEDANS, 5-[[2-[(iodoacetyl)amino]ethyl]amino]naphthalene-1sulfonic acid; Apo, apolipoprotein; CD, circular dichroism; CMC, critical micelle concentration; CR, Congo Red; DnPC, 1,2-diacyl-snglycero-3-phosphocholine, where $n=3-9$ carbon acyl chains; DHPC, dihexanoylphosphatidylcholine; FRET, fluorescence resonance energy transfer; HDL, high-density lipoprotein; LDL, low-density lipoprotein; MD, molecular dynamics; SAP, serum amyloid $P$ component; STEM, scanning transmission electron microscopy; TEM, transmission electron microscopy; ThT, thioflavin T

\section{REFERENCES}

Acharya, P., Segall, M.L., Zaiou, M., Morrow, J., Weisgraber, K.H., Phillips, M.C., Lund-Katz, S., and Snow, J. (2002). Comparison of the stabilities and unfolding pathways of human apolipoprotein $E$ isoforms by differential scanning calorimetry and circular dichroism. Biochim Biophys Acta 1584, 9-19.

Alexandrescu, A.T. (2005). Amyloid accomplices and enforcers. Protein Sci 14, 1-12.

Anantharamaiah, G.M., Hughes, T.A., Iqbal, M., Gawish, A., Neame, P.J., Medley, M.F., and Segrest, J.P. (1988). Effect of oxidation on the properties of apolipoproteins A-I and A-II. J Lipid Res 29, 309-318.

Andersen, C.B., Yagi, H., Manno, M., Martorana, V., Ban, T., Christiansen, G., Otzen, D.E., Goto, Y., and Rischel, C. (2009). Branching in amyloid fibril growth. Biophys J 96, 1529-1536.

Andreola, A., Bellotti, V., Giorgetti, S., Mangione, P., Obici, L., Stoppini, M., Torres, J., Monzani, E., Merlini, G., and Sunde, M. (2003). Conformational switching and fibrillogenesis in the amyloidogenic fragment of apolipoprotein a-I. J Biol Chem 278, 2444-2451.

Anfinsen, C.B. (1973). Principles that govern the folding of protein chains. Science 181, 223-230.

Ban, T., Hamada, D., Hasegawa, K., Naiki, H., and Goto, Y. (2003). Direct observation of amyloid fibril growth monitored by thioflavin $\mathrm{T}$ fluorescence. J Biol Chem 278, 16462-16465.

Ban, T., Hoshino, M., Takahashi, S., Hamada, D., Hasegawa, K., Naiki, H., and Goto, Y. (2004). Direct observation of Abeta amyloid fibril growth and inhibition. J Mol Biol 344, 757-767.

Ban, T., Morigaki, K., Yagi, H., Kawasaki, T., Kobayashi, A., Yuba, S., Naiki, H., and Goto, Y. (2006a). Real-time and single fibril 
observation of the formation of amyloid beta spherulitic structures. J Biol Chem 281, 33677-33683.

Ban, T., Yamaguchi, K., and Goto, Y. (2006b). Direct observation of amyloid fibril growth, propagation, and adaptation. Acc Chem Res 39, 663-670.

Benson, M.D., Liepnieks, J.J., Yazaki, M., Yamashita, T., Hamidi Asl, K., Guenther, B., and Kluve-Beckerman, B. (2001). A new human hereditary amyloidosis: the result of a stop-codon mutation in the apolipoprotein All gene. Genomics 72, 272-277.

Binger, K.J., Griffin, M.D., Heinemann, S.H., and Howlett, G.J. (2010). Methionine-oxidized amyloid fibrils are poor substrates for human methionine sulfoxide reductases A and B2. Biochemistry 49, 2981-2983.

Binger, K.J., Griffin, M.D., and Howlett, G.J. (2008a). Methionine oxidation inhibits assembly and promotes disassembly of apolipoprotein C-II amyloid fibrils. Biochemistry 47, 10208-10217.

Binger, K.J., Pham, C.L., Wilson, L.M., Bailey, M.F., Lawrence, L.J., Schuck, P., and Howlett, G.J. (2008b). Apolipoprotein C-II amyloid fibrils assemble via a reversible pathway that includes fibril breaking and rejoining. J Mol Biol 376, 1116-1129.

Booth, D.R., Tan, S.Y., Booth, S.E., Hsuan, J.J., Totty, N.F., Nguyen, O., Hutton, T., Vigushin, D.M., Tennent, G.A., Hutchinson, W.L., et al. (1995). A new apolipoprotein Al variant, Trp50Arg, causes hereditary amyloidosis. QJM 88, 695-702.

Booth, D.R., Tan, S.Y., Booth, S.E., Tennent, G.A., Hutchinson, W.L., Hsuan, J.J., Totty, N.F., Truong, O., Soutar, A.K., Hawkins, P.N., et al. (1996). Hereditary hepatic and systemic amyloidosis caused by a new deletion/insertion mutation in the apolipoprotein $\mathrm{Al}$ gene. $\mathrm{J}$ Clin Invest 97, 2714-2721.

Bosco, D.A., Fowler, D.M., Zhang, Q., Nieva, J., Powers, E.T., Wentworth, P. Jr, Lerner, R.A., and Kelly, J.W. (2006). Elevated levels of oxidized cholesterol metabolites in Lewy body disease brains accelerate alpha-synuclein fibrilization. Nat Chem Biol 2, 249-253.

Calero, M., Rostagno, A., Matsubara, E., Zlokovic, B., Frangione, B., and Ghiso, J. (2000). Apolipoprotein J (clusterin) and Alzheimer's disease. Microsc Res Tech 50, 305-315.

Castaño, E.M., Prelli, F., Pras, M., and Frangione, B. (1995). Apolipoprotein $E$ carboxyl-terminal fragments are complexed to amyloids A and L. Implications for amyloidogenesis and Alzheimer's disease. J Biol Chem 270, 17610-17615.

Cedazo-Mínguez, A., and Cowburn, R.F. (2001). Apolipoprotein E: a major piece in the Alzheimer's disease puzzle. J Cell Mol Med 5, 254-266.

Chauhan, V., Wang, X., Ramsamy, T., Milne, R.W., and Sparks, D.L. (1998). Evidence for lipid-dependent structural changes in specific domains of apolipoprotein B100. Biochemistry 37, 3735-3742.

Chisolm, G.M., and Steinberg, D. (2000). The oxidative modification hypothesis of atherogenesis: an overview. Free Radic Biol Med 28, 1815-1826.

Chiti, F., Webster, P., Taddei, N., Clark, A., Stefani, M., Ramponi, G., and Dobson, C.M. (1999). Designing conditions for in vitro formation of amyloid protofilaments and fibrils. Proc Natl Acad Sci U S A 96, 3590-3594.

Cho, H.S., Hyman, B.T., Greenberg, S.M., and Rebeck, G.W. (2001). Quantitation of apoE domains in Alzheimer disease brain suggests a role for apoE in Abeta aggregation. J Neuropathol Exp Neurol 60,
342-349.

Clark, J.I., and Muchowski, P.J. (2000). Small heat-shock proteins and their potential role in human disease. Curr Opin Struct Biol 10, 52-59.

Corder, E.H., Saunders, A.M., Strittmatter, W.J., Schmechel, D.E., Gaskell, P.C., Small, G.W., Roses, A.D., Haines, J.L., and PericakVance, M.A. (1993). Gene dose of apolipoprotein E type 4 allele and the risk of Alzheimer's disease in late onset families. Science 261, 921-923.

Damaschun, G., Damaschun, H., Fabian, H., Gast, K., Kröber, R., Wieske, M., and Zirwer, D. (2000). Conversion of yeast phosphoglycerate kinase into amyloid-like structure. Proteins 39, 204-211.

de Sousa, M.M., Vital, C., Ostler, D., Fernandes, R., Pouget-Abadie, J., Carles, D., and Saraiva, M.J. (2000). Apolipoprotein Al and transthyretin as components of amyloid fibrils in a kindred with apoAI Leu178His amyloidosis. Am J Pathol 156, 1911-1917.

Derham, B.K., and Harding, J.J. (1999). Alpha-crystallin as a molecular chaperone. Prog Retin Eye Res 18, 463-509.

Dobson, C.M. (2002). Getting out of shape. Nature 418, 729-730.

Dobson, C.M. (2003). Protein folding and misfolding. Nature 426, 884-890.

Eriksson, M., Schönland, S., Yumlu, S., Hegenbart, U., von Hutten, H., Gioeva, Z., Lohse, P., Büttner, J., Schmidt, H., and Röcken, C. (2009). Hereditary apolipoprotein Al-associated amyloidosis in surgical pathology specimens: identification of three novel mutations in the APOA1 gene. J Mol Diagn 11, 257-262.

Evans, K.C., Berger, E.P., Cho, C.G., Weisgraber, K.H., and Lansbury, P.T. Jr. (1995). Apolipoprotein $E$ is a kinetic but not a thermodynamic inhibitor of amyloid formation: implications for the pathogenesis and treatment of Alzheimer disease. Proc Natl Acad Sci U S A 92, 763-767.

Fändrich, M., Fletcher, M.A., and Dobson, C.M. (2001). Amyloid fibrils from muscle myoglobin. Nature 410, 165-166.

Frangione, B., Castaño, E.M., Wisniewski, T., Ghiso, J., Prelli, F., and Vidal, R. (1996). Apolipoprotein E and amyloidogenesis. Ciba Found Symp 199, 132-141, discussion 141-145.

Garner, B., Waldeck, A.R., Witting, P.K., Rye, K.A., and Stocker, R. (1998). Oxidation of high density lipoproteins. II. Evidence for direct reduction of lipid hydroperoxides by methionine residues of apolipoproteins Al and All. J Biol Chem 273, 6088-6095.

Genschel, J., Haas, R., Pröpsting, M.J., and Schmidt, H.H. (1998). Apolipoprotein A-I induced amyloidosis. FEBS Lett 430, 145-149.

Griffin, M.D., Mok, M.L., Wilson, L.M., Pham, C.L., Waddington, L.J., Perugini, M.A., and Howlett, G.J. (2008). Phospholipid interaction induces molecular-level polymorphism in apolipoprotein C-II amyloid fibrils via alternative assembly pathways. J Mol Biol 375, 240-256.

Gunzburg, M.J., Perugini, M.A., and Howlett, G.J. (2007). Structural basis for the recognition and cross-linking of amyloid fibrils by human apolipoprotein E. J Biol Chem 282, 35831-35841.

Häggqvist, B., Näslund, J., Sletten, K., Westermark, G.T., Mucchiano, G., Tjernberg, L.O., Nordstedt, C., Engström, U., and Westermark, P. (1999). Medin: an integral fragment of aortic smooth muscle cellproduced lactadherin forms the most common human amyloid. Proc Natl Acad Sci U S A 96, 8669-8674.

Hatters, D.M., and Howlett, G.J. (2002). The structural basis for amyloid formation by plasma apolipoproteins: a review. Eur 
Biophys J 31, 2-8.

Hatters, D.M., Lawrence, L.J., and Howlett, G.J. (2001a). Submicellar phospholipid accelerates amyloid formation by apolipoprotein C-II. FEBS Lett 494, 220-224.

Hatters, D.M., Lindner, R.A., Carver, J.A., and Howlett, G.J. (2001b). The molecular chaperone, alpha-crystallin, inhibits amyloid formation by apolipoprotein C-II. J Biol Chem 276, 33755-33761.

Hatters, D.M., MacPhee, C.E., Lawrence, L.J., Sawyer, W.H., and Howlett, G.J. (2000). Human apolipoprotein C-II forms twisted amyloid ribbons and closed loops. Biochemistry 39, 8276-8283.

Hatters, D.M., MacRaild, C.A., Daniels, R., Gosal, W.S., Thomson, N. H., Jones, J.A., Davis, J.J., MacPhee, C.E., Dobson, C.M., and Howlett, G.J. (2003). The circularization of amyloid fibrils formed by apolipoprotein C-II. Biophys J 85, 3979-3990.

Hatters, D.M., Wilson, M.R., Easterbrook-Smith, S.B., and Howlett, G. J. (2002). Suppression of apolipoprotein C-Il amyloid formation by the extracellular chaperone, clusterin. Eur J Biochem 269, 2789-2794.

Hatters, D.M., Zhong, N., Rutenber, E., and Weisgraber, K.H. (2006). Amino-terminal domain stability mediates apolipoprotein $\mathrm{E}$ aggregation into neurotoxic fibrils. J Mol Biol 361, 932-944.

Havel, R.J., Fielding, C.J., Olivecrona, T., Shore, V.G., Fielding, P.E., and Egelrud, T. (1973). Cofactor activity of protein components of human very low density lipoproteins in the hydrolysis of triglycerides by lipoproteins lipase from different sources. Biochemistry 12 , 1828-1833.

Higgins, G.A., Large, C.H., Rupniak, H.T., and Barnes, J.C. (1997). Apolipoprotein $\mathrm{E}$ and Alzheimer's disease: a review of recent studies. Pharmacol Biochem Behav 56, 675-685.

Higuchi, K., Kitagawa, K., Naiki, H., Hanada, K., Hosokawa, M., and Takeda, T. (1991a). Polymorphism of apolipoprotein A-II (apoA-II) among inbred strains of mice. Relationship between the molecular type of apoA-II and mouse senile amyloidosis. Biochem J 279, 427-433.

Higuchi, K., Naiki, H., Kitagawa, K., Hosokawa, M., and Takeda, T. (1991b). Mouse senile amyloidosis. ASSAM amyloidosis in mice presents universally as a systemic age-associated amyloidosis. Virchows Arch B Cell Pathol Incl Mol Pathol 60, 231-238.

Howlett, G.J., and Moore, K.J. (2006). Untangling the role of amyloid in atherosclerosis. Curr Opin Lipidol 17, 541-547.

Humphreys, D.T., Carver, J.A., Easterbrook-Smith, S.B., and Wilson, M.R. (1999). Clusterin has chaperone-like activity similar to that of small heat shock proteins. J Biol Chem 274, 6875-6881.

Hung, A., Griffin, M.D., Howlett, G.J., and Yarovsky, I. (2008). Effects of oxidation, $\mathrm{pH}$ and lipids on amyloidogenic peptide structure: implications for fibril formation? Eur Biophys J 38, 99-110.

Hung, A., Griffin, M.D., Howlett, G.J., and Yarovsky, I. (2009). Lipids enhance apolipoprotein C-II-derived amyloidogenic peptide oligomerization but inhibit fibril formation. J Phys Chem B 113, 9447-9453.

Kawano, M., Kawakami, M., Otsuka, M., Yashima, H., Yaginuma, T., and Ueki, A. (1995). Marked decrease of plasma apolipoprotein Al and All in Japanese patients with late-onset non-familial Alzheimer's disease. Clin Chim Acta 239, 209-211.

Kinnunen, P.K., Jackson, R.L., Smith, L.C., Gotto, A.M. Jr, and Sparrow, J.T. (1977). Activation of lipoprotein lipase by native and synthetic fragments of human plasma apolipoprotein C-II. Proc Natl Acad Sci U S A 74, 4848-4851.
Kisilevsky, R. (2000). The relation of proteoglycans, serum amyloid $P$ and apo $\mathrm{E}$ to amyloidosis current status, 2000. Amyloid 7, 23-25.

Knight, J.D., and Miranker, A.D. (2004). Phospholipid catalysis of diabetic amyloid assembly. J Mol Biol 341, 1175-1187.

Koistinaho, M., Lin, S., Wu, X., Esterman, M., Koger, D., Hanson, J., Higgs, R., Liu, F., Malkani, S., Bales, K.R., et al. (2004). Apolipoprotein $\mathrm{E}$ promotes astrocyte colocalization and degradation of deposited amyloid-beta peptides. Nat Med 10, 719-726.

Kuriyama, M., Takahashi, K., Yamano, T., Hokezu, Y., Togo, S., Osame, M., and Igakura, T. (1994). Low levels of serum apolipoprotein A I and A II in senile dementia. Jpn J Psychiatry Neurol 48, 589-593.

Lange, U., Boss, B., Teichmann, J., Klör, H.U., and Neeck, G. (2000). Serum amyloid A-an indicator of inflammation in ankylosing spondylitis. Rheumatol Int 19, 119-122.

LaRosa, J.C., Levy, R.I., Herbert, P., Lux, S.E., and Fredrickson, D.S. (1970). A specific apoprotein activator for lipoprotein lipase. Biochem Biophys Res Commun 41, 57-62.

Legge, F.S., Binger, K.J., Griffin, M.D., Howlett, G.J., Scanlon, D., Treutlein, H., and Yarovsky, I. (2009). Effect of oxidation and mutation on the conformational dynamics and fibril assembly of amyloidogenic peptides derived from apolipoprotein C-II. J Phys Chem B 113, 14006-14014.

Legge, F.S., Treutlein, H., Howlett, G.J., and Yarovsky, I. (2007). Molecular dynamics simulations of a fibrillogenic peptide derived from apolipoprotein C-II. Biophys Chem 130, 102-113.

Li, Q., Min, J., Ahn, Y.H., Namm, J., Kim, E.M., Lui, R., Kim, H.Y., Ji, Y., Wu, H., Wisniewski, T., et al. (2007). Styryl-based compounds as potential in vivo imaging agents for beta-amyloid plaques. Chembiochem 8, 1679-1687.

MacRaild, C.A., Hatters, D.M., Howlett, G.J., and Gooley, P.R. (2001). NMR structure of human apolipoprotein $C$-II in the presence of sodium dodecyl sulfate. Biochemistry 40, 5414-5421.

MacRaild, C.A., Hatters, D.M., Lawrence, L.J., and Howlett, G.J. (2003). Sedimentation velocity analysis of flexible macromolecules: self-association and tangling of amyloid fibrils. Biophys $\mathrm{J} 84$, 2562-2569.

MacRaild, C.A., Howlett, G.J., and Gooley, P.R. (2004a). The structure and interactions of human apolipoprotein C-II in dodecyl phosphocholine. Biochemistry 43, 8084-8093.

MacRaild, C.A., Stewart, C.R., Mok, Y.F., Gunzburg, M.J., Perugini, M.A., Lawrence, L.J., Tirtaatmadja, V., Cooper-White, J.J., and Howlett, G.J. (2004b). Non-fibrillar components of amyloid deposits mediate the self-association and tangling of amyloid fibrils. J Biol Chem 279, 21038-21045.

Mak, P.A., Laffitte, B.A., Desrumaux, C., Joseph, S.B., Curtiss, L.K., Mangelsdorf, D.J., Tontonoz, P., and Edwards, P.A. (2002). Regulated expression of the apolipoprotein E/C-I/C-IV/C-II gene cluster in murine and human macrophages. A critical role for nuclear liver $X$ receptors alpha and beta. J Biol Chem 277, 31900-31908.

May, P.C., and Finch, C.E. (1992). Sulfated glycoprotein 2: new relationships of this multifunctional protein to neurodegeneration. Trends Neurosci 15, 391-396.

Medeiros, L.A., Khan, T., El Khoury, J.B., Pham, C.L., Hatters, D.M., Howlett, G.J., Lopez, R., O'Brien, K.D., and Moore, K.J. (2004). Fibrillar amyloid protein present in atheroma activates CD36 signal transduction. J Biol Chem 279, 10643-10648. 
Merched, A., Xia, Y., Visvikis, S., Serot, J.M., and Siest, G. (2000). Decreased high-density lipoprotein cholesterol and serum apolipoprotein Al concentrations are highly correlated with the severity of Alzheimer's disease. Neurobiol Aging 21, 27-30.

Mok, Y.F., Ryan, T.M., Yang, S., Hatters, D.M., Howlett, G.J., and Griffin, M.D. (2010). Sedimentation velocity analysis of amyloid oligomers and fibrils using fluorescence detection. Methods. doi: 10.1016/j.ymeth.2010.10.004

Moore, K.J., El Khoury, J., Medeiros, L.A., Terada, K., Geula, C., Luster, A.D., and Freeman, M.W. (2002). A CD36-initiated signaling cascade mediates inflammatory effects of beta-amyloid. J Biol Chem 277, 47373-47379.

Mucchiano, G., Cornwell, G.G. 3rd, and Westermark, P. (1992). Senile aortic amyloid. Evidence for two distinct forms of localized deposits. Am J Pathol 140, 871-877.

Mucchiano, G.I., Häggqvist, B., Sletten, K., and Westermark, P. (2001a). Apolipoprotein A-1-derived amyloid in atherosclerotic plaques of the human aorta. J Pathol 193, 270-275.

Mucchiano, G.I., Jonasson, L., Häggqvist, B., Einarsson, E., and Westermark, P. (2001b). Apolipoprotein A-I-derived amyloid in atherosclerosis. Its association with plasma levels of apolipoprotein A-I and cholesterol. Am J Clin Pathol 115, 298-303.

Myers, S.L., Jones, S., Jahn, T.R., Morten, I.J., Tennent, G.A., Hewitt, E.W., and Radford, S.E. (2006). A systematic study of the effect of physiological factors on beta2-microglobulin amyloid formation at neutral $\mathrm{pH}$. Biochemistry 45, 2311-2321.

Naiki, H., Gejyo, F., and Nakakuki, K. (1997). Concentrationdependent inhibitory effects of apolipoprotein $E$ on Alzheimer's beta-amyloid fibril formation in vitro. Biochemistry 36, 6243-6250.

Naiki, H., Hasegawa, K., Yamaguchi, I., Nakamura, H., Gejyo, F., and Nakakuki, K. (1998). Apolipoprotein E and antioxidants have different mechanisms of inhibiting Alzheimer's beta-amyloid fibril formation in vitro. Biochemistry 37, 17882-17889.

Näslund, J., Thyberg, J., Tjernberg, L.O., Wernstedt, C., Karlström, A. R., Bogdanovic, N., Gandy, S.E., Lannfelt, L., Terenius, L., and Nordstedt, C. (1995). Characterization of stable complexes involving apolipoprotein $\mathrm{E}$ and the amyloid beta peptide in Alzheimer's disease brain. Neuron 15, 219-228.

O'Brien, K.D., Olin, K.L., Alpers, C.E., Chiu, W., Ferguson, M., Hudkins, K., Wight, T.N., and Chait, A. (1998). Comparison of apolipoprotein and proteoglycan deposits in human coronary atherosclerotic plaques: colocalization of biglycan with apolipoproteins. Circulation 98, 519-527.

Obici, L., Franceschini, G., Calabresi, L., Giorgetti, S., Stoppini, M., Merlini, G., and Bellotti, V. (2006). Structure, function and amyloidogenic propensity of apolipoprotein A-I. Amyloid 13, 191-205.

Ozawa, D., Yagi, H., Ban, T., Kameda, A., Kawakami, T., Naiki, H., and Goto, Y. (2009). Destruction of amyloid fibrils of a beta2microglobulin fragment by laser beam irradiation. J Biol Chem 284, 1009-1017.

Pepys, M.B. (2006). Amyloidosis. Annu Rev Med 57, 223-241.

Pepys, M.B., Booth, D.R., Huchinson, W.L., Gallimore, J.R., Collins, P.M., and Hohenester, E. (1997). Amyloid P component. A critical review. AMYLOID 4, 274-295.

Pepys, M.B., Herbert, J., Hutchinson, W.L., Tennent, G.A., Lachmann, H.J., Gallimore, J.R., Lovat, L.B., Bartfai, T., Alanine, A., Hertel, C., et al. (2002). Targeted pharmacological depletion of serum amyloid $\mathrm{P}$ component for treatment of human amyloidosis. Nature 417, 254-259.

Permanne, B., Perez, C., Soto, C., Frangione, B., and Wisniewski, T. (1997). Detection of apolipoprotein E/dimeric soluble amyloid beta complexes in Alzheimer's disease brain supernatants. Biochem Biophys Res Commun 240, 715-720.

Pham, C.L., Hatters, D.M., Lawrence, L.J., and Howlett, G.J. (2002). Cross-linking and amyloid formation by $\mathrm{N}$ - and $\mathrm{C}$-terminal cysteine derivatives of human apolipoprotein C-II. Biochemistry 41, 14313-14322.

Puchtler, H., and Sweat, F. (1962). Amidoblack as a stain for hemoglobin. Arch Pathol 73, 245-249.

Röcken, C., Tautenhahn, J., Bühling, F., Sachwitz, D., Vöckler, S., Goette, A., and Bürger, T. (2006). Prevalence and pathology of amyloid in atherosclerotic arteries. Arterioscler Thromb Vasc Biol 26, 676-677.

Ross, R. (1999). Atherosclerosis—an inflammatory disease. N Engl J Med 340, 115-126.

Ryan, T.M., Griffin, M.D., Teoh, C.L., Ooi, J., and Howlett, G.J. (2011). High-Affinity Amphipathic Modulators of Amyloid Fibril Nucleation and Elongation. J Mol Biol 406, 416-429

Ryan, T.M., Howlett, G.J., and Bailey, M.F. (2008). Fluorescence detection of a lipid-induced tetrameric intermediate in amyloid fibril formation by apolipoprotein C-II. J Biol Chem 283, 35118-35128.

Ryan, T.M., Teoh, C.L., Griffin, M.D., Bailey, M.F., Schuck, P., and Howlett, G.J. (2010). Phospholipids enhance nucleation but not elongation of apolipoprotein C-II amyloid fibrils. J Mol Biol 399, 731-740.

Saczynski, J.S., White, L., Peila, R.L., Rodriguez, B.L., and Launer, L. J. (2007). The relation between apolipoprotein A-I and dementia: the Honolulu-Asia aging study. Am J Epidemiol 165, 985-992.

Saunders, A.M., Strittmatter, W.J., Schmechel, D., George-Hyslop, P. H., Pericak-Vance, M.A., Joo, S.H., Rosi, B.L., Gusella, J.F., Crapper-MacLachlan, D.R., Alberts, M.J., et al. (1993). Association of apolipoprotein $\mathrm{E}$ allele epsilon 4 with late-onset familial and sporadic Alzheimer's disease. Neurology 43, 1467-1472.

Segrest, J.P., Garber, D.W., Brouillette, C.G., Harvey, S.C., and Anantharamaiah, G.M. (1994). The amphipathic alpha helix: a multifunctional structural motif in plasma apolipoproteins. Adv Protein Chem 45, 303-369.

Sipe, J.D., and Cohen, A.S. (2000). Review: history of the amyloid fibril. J Struct Biol 130, 88-98.

Soto, C., Castaño, E.M., Prelli, F., Kumar, R.A., and Baumann, M. (1995). Apolipoprotein $E$ increases the fibrillogenic potential of synthetic peptides derived from Alzheimer's, gelsolin and $A A$ amyloids. FEBS Lett 371, 110-114.

Stadtman, E.R., and Levine, R.L. (2000). Protein oxidation. Ann N Y Acad Sci 899, 191-208.

Stewart, C.R., Wilson, L.M., Zhang, Q., Pham, C.L., Waddington, L.J., Staples, M.K., Stapleton, D., Kelly, J.W., and Howlett, G.J. (2007). Oxidized cholesterol metabolites found in human atherosclerotic lesions promote apolipoprotein C-II amyloid fibril formation. Biochemistry 46, 5552-5561.

Strittmatter, W.J., Weisgraber, K.H., Huang, D.Y., Dong, L.M., Salvesen, G.S., Pericak-Vance, M., Schmechel, D., Saunders, A. M., Goldgaber, D., and Roses, A.D. (1993). Binding of human apolipoprotein $\mathrm{E}$ to synthetic amyloid beta peptide: isoform-specific 
effects and implications for late-onset Alzheimer disease. Proc Natl Acad Sci U S A 90, 8098-8102.

Tennent, G.A., Lovat, L.B., and Pepys, M.B. (1995). Serum amyloid P component prevents proteolysis of the amyloid fibrils of Alzheimer disease and systemic amyloidosis. Proc Natl Acad Sci U S A 92, 4299-4303.

Teoh, C.L., Pham, C.L., Todorova, N., Hung, A., Lincoln, C.N., Lees, E., Lam, Y.H., Binger, K.J., Thomson, N.H., Radford, S.E., et al. (2011a). A structural model for apolipoprotein C-Il amyloid fibrils: experimental characterization and molecular dynamics simulations. J Mol Biol 405, 1246-1266.

Teoh, C.L., Yagi, H., Griffin, M.D., Goto, Y., and Howlett, G.J. (2011b). Visualization of polymorphism in apolipoprotein C-Il amyloid fibrils. J Biochem 149, 67-74.

Todorova, N., Hung, A., Maaser, S.M., Griffin, M.D., Karas, J., Howlett, G.J., and Yarovsky, I. (2010). Effects of mutation on the amyloidogenic propensity of apolipoprotein C-II(60-70) peptide. Phys Chem Chem Phys 12, 14762-14774.

Westermark, G.T., Johnson, K.H., and Westermark, P. (1999). Staining methods for identification of amyloid in tissue. Methods Enzymol 309, 3-25.

Westermark, P., Mucchiano, G., Marthin, T., Johnson, K.H., and Sletten, K. (1995). Apolipoprotein A1-derived amyloid in human aortic atherosclerotic plaques. Am J Pathol 147, 1186-1192.

Wilson, L.M., Mok, Y.F., Binger, K.J., Griffin, M.D., Mertens, H.D., Lin, F., Wade, J.D., Gooley, P.R., and Howlett, G.J. (2007). A structural core within apolipoprotein C-II amyloid fibrils identified using hydrogen exchange and proteolysis. J Mol Biol 366, 1639-1651. Wisniewski, T., Lalowski, M., Golabek, A., Vogel, T., and Frangione, B. (1995). Is Alzheimer's disease an apolipoprotein E amyloidosis? Lancet 345, 956-958.

Wong, Y.Q., Binger, K.J., Howlett, G.J., and Griffin, M.D. (2010). Methionine oxidation induces amyloid fibril formation by full-length apolipoprotein A-I. Proc Natl Acad Sci U S A 107, 1977-1982.

Yagi, H., Ban, T., Morigaki, K., Naiki, H., and Goto, Y. (2007). Visualization and classification of amyloid beta supramolecular assemblies. Biochemistry 46, 15009-15017.

Yagi, H., Ozawa, D., Sakurai, K., Kawakami, T., Kuyama, H., Nishimura, O., Shimanouchi, T., Kuboi, R., Naiki, H., and Goto, Y. (2010). Laser-induced propagation and destruction of amyloid beta fibrils. J Biol Chem 285, 19660-19667.

Zhang, Q., Powers, E.T., Nieva, J., Huff, M.E., Dendle, M.A., Bieschke, J., Glabe, C.G., Eschenmoser, A., Wentworth, P. Jr, Lerner, R.A., et al. (2004). Metabolite-initiated protein misfolding may trigger Alzheimer's disease. Proc Natl Acad Sci U S A 101, 4752-4757.

Zhao, H., Tuominen, E.K., and Kinnunen, P.K. (2004). Formation of amyloid fibers triggered by phosphatidylserine-containing membranes. Biochemistry 43, 10302-10307.

Zheng, L., Nukuna, B., Brennan, M.L., Sun, M., Goormastic, M., Settle, M., Schmitt, D., Fu, X., Thomson, L., Fox, P.L., et al. (2004). Apolipoprotein A-I is a selective target for myeloperoxidasecatalyzed oxidation and functional impairment in subjects with cardiovascular disease. J Clin Invest 114, 529-541. 